\title{
Retropubic, laparoscopic and mini-laparoscopic radical prostatectomy: a prospective assessment of patient scar satisfaction
}

\author{
Carmelo Quattrone - Antonio Cicione - Carlos Oliveira - Riccardo Autorino • \\ Francesco Cantiello $\cdot$ Vincenzo Mirone $\cdot$ Marco De Sio $\cdot$ Luca Carrubbo • \\ Rocco Damiano $\cdot$ Carlo Pavone $\cdot$ Estevão Lima
}

Received: 18 June 2014 / Accepted: 19 October 2014

(C) Springer-Verlag Berlin Heidelberg 2014

\begin{abstract}
Purpose To compare patient scar satisfaction after retropubic, standard laparoscopic, mini-laparoscopic (ML) and open radical prostatectomy (RP).

Methods Patients undergoing RP for a diagnosis of localized prostate cancer at a single academic hospital between September 2012 and December 2013 were enrolled in this prospective nonrandomized study. The patients were included in three study arms: open surgery, VLP and ML. A skin stapler was used for surgical wound closure in all cases. Demographic and main surgical outcomes, including perioperative complications, were analyzed. Surgical scar satisfaction was measured using the Patient and Observer Scar Assessment Questionnaire (POSAS) and the two Body
\end{abstract}

C. Quattrone · C. Pavone

Department of Urology, University of Palermo, Palermo, Italy

A. Cicione $\cdot$ F. Cantiello $\cdot$ R. Damiano

Department of Urology, Magna Graecia University, Catanzaro, Italy

C. Oliveira $\cdot$ E. Lima

Department of Urology, Braga Hospital, Braga, Portugal

R. Autorino $\cdot$ M. De Sio

Urology Unit, Second University of Naples, Naples, Italy

R. Autorino ( $\square)$

Urology Institute, University Hospitals, Case Western Reserve

University, Cleveland, OH, USA

e-mail: ricautor@gmail.com

V. Mirone

Department of Urology, Federico II University, Naples, Italy

L. Carrubbo

Department of Economy and Law, University of Cassino

and Southern Lazio, Cassino, Italy
Image Questionnaire (BIQ) scales, respectively, recorded at skin clips removal and either at 6 months after surgery.

Results Overall, 32 patients were enrolled and completed the 6 month of follow-up. At clips removal, laparoscopic approaches offered better scar result than open surgery according to the POSAS. However, at 6 months, no differences were detected between VLP and open, whereas ML was still associated with a better scar outcome $(p=0.001)$. This finding was also confirmed by both BIQ scales, including the body image score (ML $9.8 \pm 1.69$, open $15.73 \pm 3.47$, VLP $13.27 \pm 3.64$; $p=0.001$ ) and the cosmetic score (ML $16.6 \pm 4.12$, open $10 \pm 1.9, \mathrm{LP} 12.91 \pm 3.59 ; p=0.001)$. Small sample size and lack of randomization represent the main limitations of this study.

Conclusions ML RP offers a better cosmetic outcome when compared to both open and standard laparoscopic $\mathrm{RP}$, representing a step toward minimal surgical scar. The impact of scar outcome on RP patients' quality of life remains to be determined.

Keywords Mini-laparoscopy · Radical prostatectomy · POSAS $\cdot$ Surgical scar

\section{Introduction}

Radical prostatectomy (RP) represents the standard surgical treatment for patients with low- and intermediate-risk localized PCa and a life expectancy $>10$ years [1]. Open RP has represented the gold standard technique for over 30 years, and it is still routinely performed [2]. The laparoscopic [3] and, more recently, the robot-assisted approach [4] have been largely adopted with the aim of reducing surgical morbidity. 
Over the last 5 years, the field of urologic surgery has witnessed an increasing interest toward scarless techniques, with the ultimate aim of further lowering surgical morbidity and expediting postoperative recovery. Based on this concept, novel surgical approaches such as natural orifice translumenal endoscopic surgery (NOTES) and laparoendoscopic single-site surgery (LESS) have been explored [5]. However, these techniques carry some limitations, including an unfavorable ergonomy and the lack of optimal instrumentation, are still under development.

On the other hand, mini-laparoscopy (ML), which was initially conceived in the early $90 \mathrm{~s}$ maintaining the key principles of standard laparoscopy (i.e., triangulation), has been rediscovered thanks to the recent availability of more reliable instrumentation [6]. In terms of surgical scarring, the use of trocars of smaller diameter, not requiring formal skin closure, could potentially translate into less pain. A broad range of common urological procedures have safely and effectively performed with this newly re-discovered technique, mostly for kidney diseases [7]. Porpiglia et al. recently showed a better patients scar satisfaction using ML instruments to carry out laparoscopic pyeloplasty [8].

Despite the large number of studies comparing oncological and functional outcomes between the different surgical approaches $[9,10]$, available literature on objective scar assessment of patients undergoing RP remains anecdotal [11].

The aim of present study was to analyze the cosmetic result of ML RP compared with open and standard laparoscopic approaches.

\section{Patients and methods}

Study design

This is a prospective nonrandomized study carried out in a tertiary care institution (Braga Hospital, Braga, Portugal) between September 2012 and December 2013. The study was conducted in accordance with good clinical practice guidelines and the Declaration of Helsinki, and the local institutional review board approved the protocol. All subjects gave written informed consent before enrollment in the study. Patients were allocated in 3 study groups according to surgeon preference: open surgery, standard laparoscopy and ML.

\section{Patients}

Eligible patients had a diagnosis of localized prostate cancer with a PSA level $\leq 10 \mathrm{ng} / \mathrm{ml}$, biopsy Gleason score $\leq 7$, age $\leq 70$ years, trans-rectal ultrasound prostate volume estimated $\leq 50 \mathrm{cc}$, life expectative $\geq 10$ years and American
Society of Anesthesiology score (ASA score) $\leq 2$. Exclusion criteria were previous pelvic surgery or trans-urethral resection of the prostate (TURP), diabetes mellitus, chronic use of corticosteroid medication.

Surgical techniques

All procedures were performed by a senior surgeon with extensive laparoscopic experience (E.L.). For the open approach, a standard retropubic technique through an infraumbilical midline incision was used [12]. For both laparoscopic and ML approaches, the same surgical technique was adopted [13]. A limited pelvic lymphadenectomy was selectively performed in high-risk (Gleason score $>6$ ) patients. In brief, the patient was placed in Trendelenburg supine position (Fig. 1). A 10-mm skin incision was made laterally to umbilical scar in order to create a preperitoneal space by introducing a dissecting balloon trocar. After that, the balloon trocar was replaced by a $12-\mathrm{mm}$ optical trocar. Two $3.5-\mathrm{mm}$ trocars $(5 \mathrm{~mm}$ in case of standard laparoscopy) were positioned medially to the anterior superior iliac spine, bilaterally. One 5-mm trocar was placed approximately 3 fingerbreadths medially to the left anterior superior iliac spine while another $3.5-\mathrm{mm}(5 \mathrm{~mm}$ in case of standard laparoscopy) trocar in the right para-rectal line, between the optical trocar and the previously placed right 3.5-mm trocar. In all procedures, sterile skin closure clips were used and removed at postoperative day 7 (Fig. 2). Protocol for pain management was the same for all patients regardless the technique. Patients received intravenous ketorolac for $48 \mathrm{~h}$. In addition, tramadol was given "on demand" for severe pain.

\section{Outcomes}

Baseline demographic, surgical outcomes, intra (graded according to Satava) [14]- and postoperative complications (graded according to Clavien-Dindo) [14]), and pathology findings were recorded. Biochemical recurrence was defined according to current guidelines as single PSA $>0.2 \mathrm{ng} / \mathrm{ml}$ followed by a subsequent rise. Functional outcomes related to continence (number of pads per day) and erectile function (IIEF score) were recorded. Scar evaluation and patient scar satisfaction represented the primary outcomes. The first one was performed at time of clips removal and at 6 month after surgery. At both times, a validated assessment tool, the Patient and Observer Scar Assessment Questionnaire (POSAS), was used [15, 16]). This consists of two scales with six items rating 1-10 where the highest score reflects the worst finding. The observer items were collected by an "observer" (a physician part of the research team). Patient scar satisfaction was assessed using the Body Image Questionnaire (BIQ) [17] at 


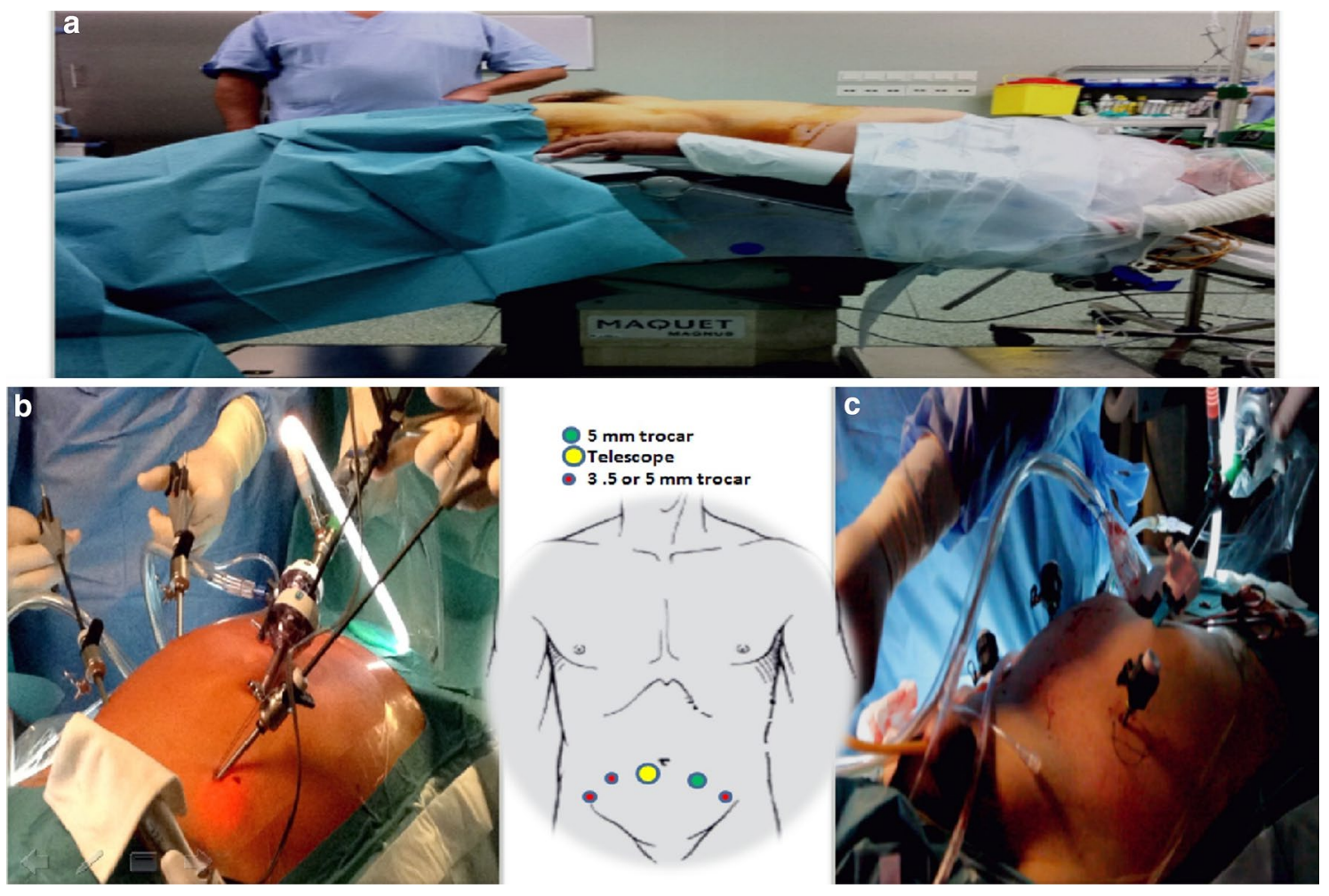

Fig. 1 Patient position (a) and trocar placement for standard laparoscopy (b) and mini-laparoscopic (c) radical prostatectomy
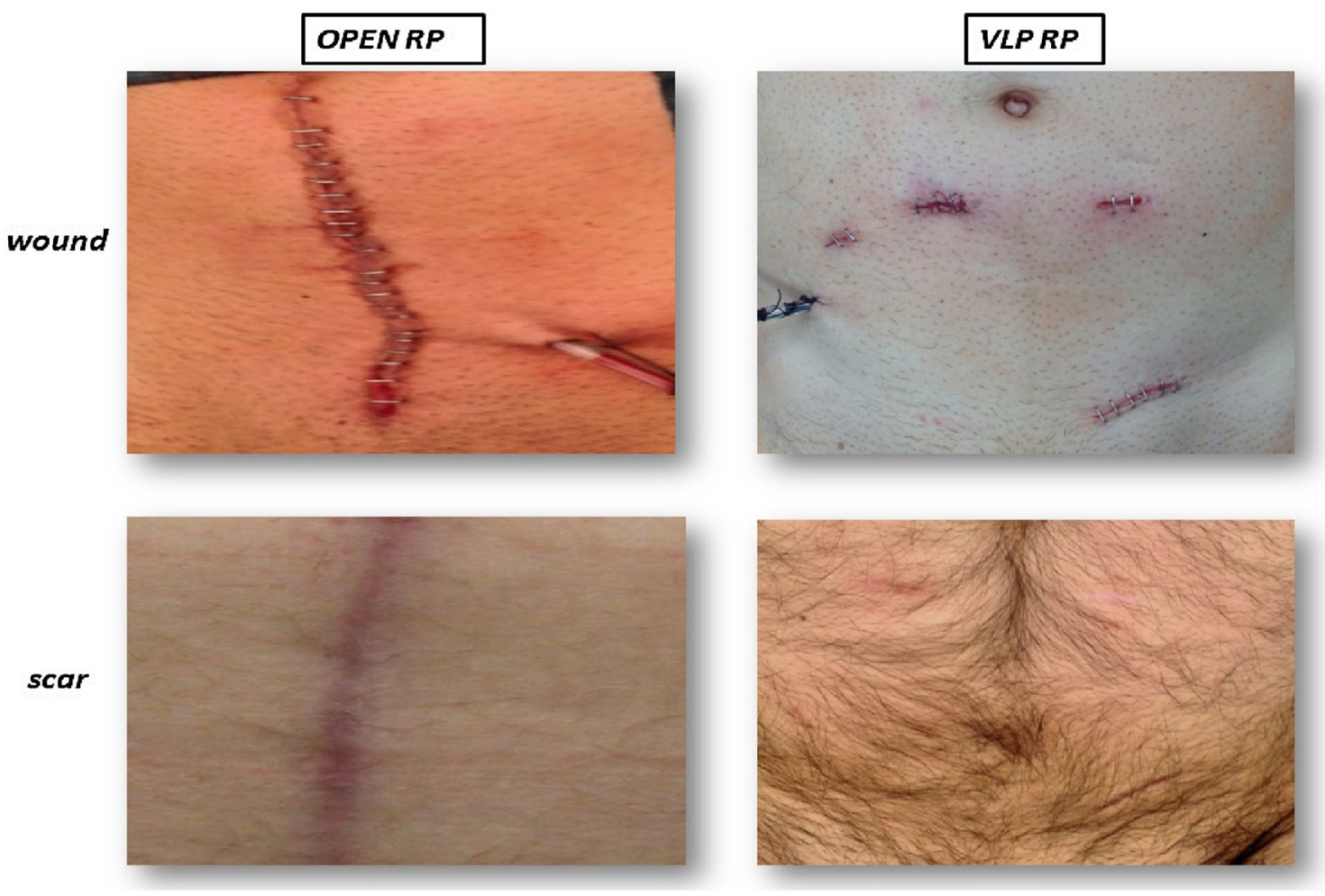

Fig. 2 Surgical wound and scar at 6 months after open or laparoscopic approaches 
Table 1 Demographics

\begin{tabular}{lllll}
\hline & $\begin{array}{l}\text { Open } \\
(n=11)\end{array}$ & $\begin{array}{l}\text { VLP } \\
(n=10)\end{array}$ & $\begin{array}{l}\text { ML } \\
(n=11)\end{array}$ & $p$ value \\
\hline Age, years & $63.2 \pm 3.4$ & $67.1 \pm 2.9$ & $64.4 \pm 4.8$ & $\mathrm{~ns}$ \\
BMI, kg/m² & $25.1 \pm 2.1$ & $23.6 \pm 1.2$ & $24.7 \pm 0.9$ & $\mathrm{~ns}$ \\
ASA score & & & & \\
1 & $8(72.7)$ & $8(80)$ & $9(81.8)$ & $\mathrm{ns}$ \\
2 & $3(27.3)$ & $2(20)$ & $2(18.2)$ & \\
Prostate vol- & $37.1 \pm 8.3$ & $38.3 \pm 5.4$ & $44.5 \pm 2.3$ & $\mathrm{~ns}$ \\
ume, cc & & & & \\
PSA, ng/dl & $6.05 \pm 2.7$ & $5.89 \pm 1.6$ & $5.65 \pm 1.7$ & $\mathrm{~ns}$ \\
Biopsy Gleason score & & & \\
Score 6 & $7(63.6)$ & $9(90)$ & $9(81.8)$ & $\mathrm{ns}$ \\
Score 7 & $4(36.4)$ & $1(10)$ & $2(18.2)$ & \\
Baseline erectile function & & & \\
IEEF score & $21.1(2.7)$ & $21.2(2.9)$ & $20.9(2.8)$ & $\mathrm{ns}$ \\
No/mild ED & $11(100)$ & $10(100)$ & $11(100)$ & \\
Moderate/ & $0(0)$ & $0(0)$ & $0(0)$ & \\
\multicolumn{2}{l}{ severe ED } & & &
\end{tabular}

Values expressed as mean $\pm(\mathrm{SD})$ or $n(\%)$

$\mathrm{ns}=$ nonsignificant $(p>0.05)$ for all pairwise comparisons

$V L P$ video laparoscopy, $M L$ mini-laparoscopy, $B M I$ body mass index, $P S A$ prostate specific antigen, $E D$ erectile dysfunction

${ }^{\wedge}$ Based on IIEF (International Index Erectile Function): 22-25: no erectile dysfunction; 17-21: mild erectile dysfunction; 12-16: mildto-moderate erectile dysfunction; 8-11: moderate erectile dysfunction; 5-7: severe erectile dysfunction

6 months after surgery. This is composed of two spheres: five questions about operation impact on body image (overall range 5-low to 20-high impact) and three questions about satisfaction on scar appearance (overall range 3-very unsatisfied to 24-very satisfied). A common postoperative protocol in terms of analgesia was employed. A visual analog pain score (VAPS) at postoperative day 1 and at time of discharge was used to measure surgical pain.

\section{Statistical analysis}

The statistical significance of differences in means and proportions was tested with one-way ANOVA test followed by Bonferroni post hoc test and the Pearson $\chi^{2}$ test, respectively. Statistical significance was set at $p<0.05$. All data were analyzed using Statistical Package for Social Sciences (SPSS) 17.0 for Windows.

\section{Results}

Overall, 32 patients were enrolled and completed the 6-month follow-up. Eleven patients underwent to open RP, ten to standard laparoscopic RP, and eleven to ML RP. No significant differences were detected between the groups in terms of baseline demographics (Table 1).

Surgical outcomes, pathology findings and functional outcomes are summarized in Table 2. Only one (9\%) Satava grade 1 intraoperative complication occurred during a ML RP. Postoperative complications were mostly Clavien grade 1 . On pathology, the disease was mostly organ-confined low-grade cancer and negative surgical margins. No patient developed biochemical recurrence at 6 months after surgery. There was no difference in terms of 6-month functional outcomes between groups.

Table 3 summarizes analgesic use, pain assessment, scar evaluation and patient scar satisfaction. Both laparoscopic approaches reported a lower VAPS than open surgery at postoperative day $1(p=0.01)$ and at patients discharge $(p=0.006)$. Moreover, a lower (nonstatistically significant) use of in-hospital "on-demand" tramadol was recorded for minimally invasive techniques compared to open $(p=0.1)$. Scar assessment was deemed to be better at clips removal for laparoscopic approaches compared to open, by the observer $(p=0.001)$ and the patient $(p=0.001)$. At 6 months, according to the observer assessment, standard laparoscopy and mini-laparoscopy were still both better than open $(p=0.001)$, whereas ML performed better than standard lap and open according to the patient $(p=0.001)$.

This finding was also confirmed by BIQ assessment at 6 months when patients in the ML group scored higher than those of open and standard laparoscopy group for both body image score $(p=0.001)$ and cosmetic score $(p=0.001)$.

\section{Discussion}

Assessment of surgical scars represents a challenging task as both patient-related factors, such as age and concomitant disease, and tissue-related features (blood supply, infection) affect healing of surgical wounds. Thus, investigators are still making several attempts to devise a reliable and valid scar assessment scale in a broad range of scar types [15].

The use of smaller laparoscopic instruments (i.e., minilaparoscopy) has been recently rediscovered with the purpose to minimize patient morbidity and obtain a scarless surgery preserving the principles of standard laparoscopy, above all instrument triangulation. A variety of urological procedures have been successfully performed using ML, including pyeloplasty, radical nephrectomy, radical prostatectomy and adrenalectomy [7].

For most patients contemplating urological surgery, cosmesis is of less concern than surgeon reputation and avoidance of surgical complications [18]. The aim of the present study was to compare three currently available surgical 
Table 2 Surgical outcomes, pathology findings and functional outcomes
Values expressed as mean $\pm(\mathrm{SD})$ or $n(\%)$

$\mathrm{ns}=$ nonsignificant $(p>0.05)$ for all pairwise comparison

Intraoperative complications:

Satava grade $1=$ substitution

of a $3 \mathrm{~mm}$ with a $5-\mathrm{mm}$ trocar;

Satava grade $2=$ conversion

to open. Postoperative

complications: Clavien Grade

$1=$ fever

$V L P$ video laparoscopy, $M L$ mini-laparoscopy, $E D$ erectile dysfunction, GS Gleason score

${ }^{\wedge}$ Based on IIEF (International Index Erectile Function):

22-25: no erectile dysfunction;

17-21: mild erectile

dysfunction; 12-16: mild-to-

moderate erectile dysfunction;

8-11: moderate erectile

dysfunction; 5-7: severe erectile

dysfunction

\begin{tabular}{|c|c|c|c|c|}
\hline & Open $(n=11)$ & $\operatorname{VLP}(n=10)$ & $\operatorname{ML}(n=11)$ & $p$ value \\
\hline \multicolumn{5}{|l|}{ Surgical outcomes } \\
\hline Operative time, $\min$ & $110.30 \pm 18.50$ & $124.60 \pm 15.70$ & $118.69 \pm 25.20$ & ns \\
\hline Hospital stay, days & $4.02 \pm 1.50$ & $3.89 \pm 1.23$ & $3.79 \pm 1.51$ & ns \\
\hline \multicolumn{5}{|l|}{ Intraop. complications } \\
\hline Satava grade 1 & 0 & 0 & $1(9)$ & ns \\
\hline Satava grade 2 & 0 & 0 & 0 & \\
\hline \multicolumn{5}{|l|}{ Postop. complications } \\
\hline Clavien grade 1 & $2(18.1)$ & $1(10)$ & $1(9)$ & ns \\
\hline Clavien grade 2 & 0 & 0 & 0 & \\
\hline Time to catheter removal, days & $10 \pm 2.51$ & $10 \pm 1.31$ & $10 \pm 1.50$ & ns \\
\hline \multicolumn{5}{|l|}{ Pathology findings } \\
\hline \multicolumn{5}{|l|}{ pStage } \\
\hline $\mathrm{T} 2 \mathrm{~b}$ & $1(9)$ & $2(20)$ & $1(9)$ & $\mathrm{ns}$ \\
\hline $\mathrm{T} 2 \mathrm{c}$ & $9(82)$ & $8(80)$ & $9(82)$ & \\
\hline $\mathrm{T} 3 \mathrm{a}$ & $1(9)$ & 0 & $1(9)$ & \\
\hline \multicolumn{5}{|l|}{ Gleason score } \\
\hline 6 & $7(63.3)$ & $7(70)$ & $8(72.7)$ & ns \\
\hline 7 & $4(36.4)$ & $3(30)$ & $3(27.3)$ & \\
\hline Positive surgical margins & $3(27.2)$ & $2(20)$ & $2(18.2)$ & ns \\
\hline \multicolumn{5}{|l|}{ 12-month functional outcomes } \\
\hline \multicolumn{5}{|l|}{ Continence } \\
\hline $0-1 \mathrm{pad}$ & $8(91)$ & $9(90)$ & $11(100)$ & ns \\
\hline$\geq 2$ pads & $1(9)$ & $1(10)$ & $0(0)$ & \\
\hline \multicolumn{5}{|l|}{ Erectile function ${ }^{\wedge}$} \\
\hline IIEF score & $12.9(3.1)$ & $14.5(2.6)$ & $14.2(3.1)$ & ns \\
\hline No/mild ED & $7(64)$ & $9(90)$ & $9(82)$ & \\
\hline Moderate/severe ED & $4(36)$ & $1(10)$ & $2(18)$ & \\
\hline
\end{tabular}

Table 3 Analgesic use, pain assessment and cosmetic outcomes in the three study groups

\begin{tabular}{|c|c|c|c|c|}
\hline & Open $(n=11)$ & Standard VLP $(n=10)$ & $\operatorname{ML}(n=11)$ & $p$ value \\
\hline Use of "on-demand" tramadol, mg & $40 \pm(51)$ & $9 \pm(30)$ & $9 \pm(30)$ & ns \\
\hline \multicolumn{5}{|l|}{ VAPS } \\
\hline Postop. day 1 & $2.82 \pm 0.75$ & $2.02 \pm 0.89 *$ & $1.72 \pm 0.82 *$ & $0.01 *$ \\
\hline Discharge & $2.09 \pm 0.7$ & $1.18 \pm 0.87 *$ & $0.90 \pm 0.88^{*}$ & $0.006^{*}$ \\
\hline \multicolumn{5}{|l|}{ POSAS at clips removal } \\
\hline Observer & $18.36 \pm 3.44$ & $10.64 \pm 4.03$ & $8.4 \pm 2.22$ & $0.001 *$ \\
\hline Patient & $23.64 \pm 6.19$ & $15.91 \pm 4.85$ & $10.5 \pm 3.24$ & $0.001 *$ \\
\hline \multicolumn{5}{|l|}{ POSAS at 6 months } \\
\hline Observer & $14.73 \pm 3.50$ & $9.09 \pm 3.48$ & $7.5 \pm 8.10$ & $0.001 *$ \\
\hline Patient & $18.01 \pm 4.92$ & $14.64 \pm 3.17$ & $8.10 \pm 2.23$ & $0.001^{\wedge}$ \\
\hline \multicolumn{5}{|l|}{ BIQ at 6 months } \\
\hline Body image score & $15.73 \pm 3.47$ & $13.27 \pm 3.64$ & $9.8 \pm 1.69$ & $0.001^{\wedge}$ \\
\hline Cosmetic score & $10 \pm 1.9$ & $12.91 \pm 3.59$ & $16.6 \pm 4.12$ & $0.001^{\wedge}$ \\
\hline
\end{tabular}

Values expressed as mean $\pm \mathrm{SD}$

$\mathrm{ns}=$ nonsignificant $(p>0.05)$ for all pairwise comparisons

VAPS visual analog pain score, POSAS Patient and Observer Scar Assessment Questionnaire, BIQ Body Image Questionnaire, $M L$ mini-laparoscopy, $V L P$ video laparoscopy

* $p$ significant for pairwise comparison between ML-Open and standard VLP-Open (nonsignificant for other comparisons)

$\wedge p$ significant for pairwise comparison between ML-Open and ML-VLP (nonsignificant for other comparisons) 
approaches for RP in terms of surgical scars and their perception by the patients. With the recent interest in scarless techniques in urology, this comparative assessment can be regarded as an attempt to better understand how patients undergoing this procedure perceive the impact of surgical scars on their body image. Bucher reported as patients are usually fascinated by new surgical approaches in particular when surgical risk is similar to traditional approach and a scar-free surgery may be achieved [19].

Interestingly, our findings suggest that at time of suture removal ( 7 days after surgery in the present study), both patient and physician have a better perception of scar related to laparoscopic approaches compared to those related to open surgery. However, at 6 months after surgery, this finding is only confirmed by physician while from a patient' point of view standard laparoscopy and open approach perform similarly, with only mini-laparoscopy holding a better scar assessment. These findings might be explained by fact that scarring is a time-dependent process [20] and cosmetic satisfaction may be fluid [21].

Delongchamps et al. [11] also found no significant difference neither in patient's scar satisfaction nor in scar-related impact on quality of life between open and standard laparoscopic RP at 3 months postoperatively. In particular, using a nonvalidated questionnaire to compute the impact of scar on quality of life (where " 0 " as no impact and " 10 " as the highest impact), the median reported values were 0 and 1 , respectively, for patients underwent to open and laparoscopic RP.

In a recent review on public perception of scarless surgery, Autorino et al. [5] showed that safety and efficacy are as the primary surgical outcomes for patients while scar issue is such significant just when equivalence results and risks are assured. In a prospective comparative study, Porpiglia et al. showed that patients who underwent minilaparoscopic pyeloplasty were significantly more satisfied with their cosmetic result than those who had standard laparoscopic approach [8]. To note, this finding was also obtained using the POSAS Questionnaire. A better cosmetic outcome using smaller trocar has also been reported in general surgery literature thought comparative studies on standard laparoscopic cholecystectomy [22, 23]. Matching these findings with ours and those ones of Delongchamps [11], one can speculate that cosmetic result is a secondary concern for patient undergone to oncological surgery and it should be considered as primary just when oncological and functional outcomes are comparable.

A common postoperative analgesia protocol was used for all study groups. A lower use of "on-demand" analgesics was recorded for VLP and ML compared to open, without reaching a statistical significance. There were no differences in terms of VAPS between the two laparoscopic approaches. A recent systematic review looking at this same parameter in patients undergoing cholecystectomy concluded that ML seems to provide a lower degree of pain when compared to standard laparoscopy [24]. This may be explained by the fact that our ML-RP was not a "pure" 3-mm surgery. This is due to the drawbacks of currently available $3 \mathrm{~mm}$ instrumentation, such as the absence of $3 \mathrm{~mm} \mathrm{Hem}$-o-lok applicator and the low resolution of $3 \mathrm{~mm}$ telescope.

Some study limitations should be recognized. The sample size in each of the study arms is limited. As this was conceived as an exploratory study, a formal sample size calculation was not performed. However, the prospective study design, despite the lack of randomization, represents a notable study feature. The short length of follow-up and the lack of quality of life assessment can also be regarded as major limitations. More robust analysis has been already performed by others about surgical, functional and oncological outcomes for different radical prostatectomy approaches $[9,10,25]$. The lack of a quality of life assessment does not allow determining how the cosmetic result impact this in oncological patients.

Despite these limitations, to the best of our best knowledge, this represents the first prospective study assessing these three approaches for RP using validated assessment tools (i.e., POSAS, BIQ, VAPS) and our results indicate that ML approach might offer a better surgical scar from the patient's point of view. Thus, further studies are warranted in this field.

\section{Conclusions}

ML can be regarded as an emerging minimally invasive surgical option in patients undergoing RP. Findings from the present prospective study suggest that ML can offer a better cosmetic outcome when compared to both open and standard laparoscopy, representing a significant step toward minimal surgical scar and less surgical pain. How these factors impact the overall quality of life of this patient population remains to be addressed.

Conflict of interest The authors declare that they have no conflict of interest.

Ethical standard This study was approved by our ethics committee and performed in accordance with the ethical standards laid down in the 1964 Declaration of Helsinki and its later amendments. All persons gave their informed consent prior to their inclusion in the study.

\section{References}

1. Heidenreich A, Bastian PJ, Bellmunt J, Bolla M, Joniau S, van der Kwast T, Mason M, Matveev V, Wiegel T, Zattoni F, Mottet N (2014) EAU guidelines on prostate cancer. part 1: screening, 
diagnosis, and local treatment with curative intent-update 2013. Eur Urol 65:124-137

2. Mullins JK, Feng Z, Trock BJ, Epstein JI, Walsh PC, Loeb S (2012) The impact of anatomical radical retropubic prostatectomy on cancer control: the 30-year anniversary. J Urol 188:2219-2224

3. Hruza M, Bermejo JL, Flinspach B, Schulze M, Teber D, Rumpelt HJ, Rassweiler JJ (2013) Long-term oncological outcomes after laparoscopic radical prostatectomy. BJU Int 111:271-280

4. Montorsi F, Wilson TG, Rosen RC, Ahlering TE, Artibani W, Carroll PR, Costello A, Eastham JA, Ficarra V, Guazzoni G, Menon M, Novara G, Patel VR, Stolzenburg JU, Van der Poel H, Van PH, Mottrie A (2012) Best practices in robot-assisted radical prostatectomy: recommendations of the Pasadena Consensus Panel. Eur Urol 62:368-381

5. Autorino R, White WM, Gettman MT, Khalifeh A, De SM, Lima E, Kaouk JH (2012) Public perception of "scarless" surgery: a critical analysis of the literature. Urology 80:495-502

6. Pini G, Rassweiler J (2012) Minilaparoscopy and laparoendoscopic single-site surgery: mini- and single-scar in urology. Minim Invasive Ther Allied Technol 21:8-25

7. Porpiglia F, Autorino R, Cicione A, Pagliarulo V, Falsaperla M, Volpe A et al (2014) Contemporary urologic minilaparoscopy: indications, techniques and surgical outcomes in a multi-institutional European cohort. J Endourol 28(8):951-957

8. Fiori C, Morra I, Bertolo R, Mele F, Chiarissi ML, Porpiglia F (2013) Standard vs mini-laparoscopic pyeloplasty: perioperative outcomes and cosmetic results. BJU Int 111:E121-E126

9. Robertson C, Close A, Fraser C, Gurung T, Jia X, Sharma P, Vale L, Ramsay C, Pickard R (2013) Relative effectiveness of robotassisted and standard laparoscopic prostatectomy as alternatives to open radical prostatectomy for treatment of localised prostate cancer: a systematic review and mixed treatment comparison meta-analysis. BJU Int 112:798-812

10. Moran PS, O'Neill M, Teljeur C, Flattery M, Murphy LA, Smyth G, Ryan M (2013) Robot-assisted radical prostatectomy compared with open and laparoscopic approaches: a systematic review and meta-analysis. Int J Urol 20:312-321

11. Delongchamps NB, Belas O, Saighi D, Zerbib M, Peyromaure M (2013) Prospective comparison of scar-related satisfaction and quality of life after laparoscopic versus open radical prostatectomy: no differences from patients' point of view. World J Urol 31:389-393

12. Walsh PC (1998) Anatomic radical prostatectomy: evolution of the surgical technique. J Urol 160:2418-2424

13. Stolzenburg JU, Kallidonis P, Minh D, Dietel A, Hafner T, Dimitriou D, Al-Aown A, Kyriazis I, Liatsikos EN (2009) Endoscopic extraperitoneal radical prostatectomy: evolution of the technique and experience with 2400 cases. J Endourol 23:1467-1472

14. Kazaryan AM, Rosok BI, Edwin B (2013) Morbidity assessment in surgery: refinement proposal based on a concept of perioperative adverse events. ISRN Surg 2013:625093

15. Durani P, McGrouther DA, Ferguson MW (2009) Current scales for assessing human scarring: a review. J Plast Reconstr Aesthet Surg 62:713-720

16. http://www.posas.org. Patient and Observer Scar Assessment Scale (2014)

17. Dunker MS, Stiggelbout AM, van Hogezand RA, Ringers J, Griffioen G, Bemelman WA (1998) Cosmesis and body image after laparoscopic-assisted and open ileocolic resection for Crohn's disease. Surg Endosc 12:1334-1340

18. Olweny EO, Mir SA, Best SL, Park SK, Donnally IC, Cadeddu JA, Tracy CR (2012) Importance of cosmesis to patients undergoing renal surgery: a comparison of laparoendoscopic single-site (LESS), laparoscopic and open surgery. BJU Int 110:268-272

19. Bucher P, Pugin F, Ostermann S, Ris F, Chilcott M, Morel P (2011) Population perception of surgical safety and body image trauma: a plea for scarless surgery ? Surg Endosc 25:408-415

20. Linares HA (1996) From wound to scar. Burns 22:339-352

21. Golkar FC, Ross SB, Sperry S, Vice M, Luberice K, Donn N, Morton C, Hernandez JM, Rosemurgy AS (2012) Patients' perceptions of laparoendoscopic single-site surgery: the cosmetic effect. Am J Surg 204:751-761

22. Bisgaard T, Klarskov B, Trap R, Kehlet H, Rosenberg J (2002) Microlaparoscopic vs conventional laparoscopic cholecystectomy: a prospective randomized double-blind trial. Surg Endosc 16:458-464

23. Novitsky YW, Kercher KW, Czerniach DR, Kaban GK, Khera S, Gallagher-Dorval KA, Callery MP, Litwin DE, Kelly JJ (2005) Advantages of mini-laparoscopic vs conventional laparoscopic cholecystectomy: results of a prospective randomized trial. Arch Surg 140:1178-1183

24. Gurusamy KS, Samraj K, Ramamoorthy R, Farouk M, Fusai G, Davidson BR (2010) Miniport versus standard ports for laparoscopic cholecystectomy. Cochrane Database Syst Rev CD006804

25. Ficarra V, Novara G, Artibani W, Cestari A, Galfano A, Graefen M, Guazzoni G, Guillonneau B, Menon M, Montorsi F, Patel V, Rassweiler J, Van PH (2009) Retropubic, laparoscopic, and robotassisted radical prostatectomy: a systematic review and cumulative analysis of comparative studies. Eur Urol 55:1037-1063 